\title{
Coverage Holes Detecting and Healing in Hybrid Wireless Networks
}

\author{
Chengsi Qi, Guanglin Zhang ${ }^{\S}$, Demin Li and Jiajie Ren \\ College of Information Science and Technology and Engineering Research \\ Center of Fashion Technology Digitized Textile Ministry of Education, \\ Donghua University, Shanghai, 201620, China \\ \{chengsiqi, renjiajie\}@mail.dhu.edu.cn, \{glzhang,deminli\}@dhu.edu.cn \\ $\S$ Corresponding author: Guanglin Zhang
}

\begin{abstract}
Nowadays, wireless sensor network which consists of numerous tiny sensors has been widely used. One of the major challenges in such networks is how to cover the sensing area effectively and maintain longer network lifetime with limited energy simultaneously. In this paper, we study hybrid network which contains both static and mobile sensors. We divide monitoring area into Delaunay Triangulation (DT) by using of Delaunay theory, based on the DT we estimate static sensors coverage holes and then work out the position of assisted mobile nodes in each triangle. In addition, we simulate our algorithm and compared with the previous methods on the platform MATLAB. The results show our algorithm is simpler and more efficient than the previous.
\end{abstract}

\section{CCS CONCEPTS}

- Computer systems organization $\rightarrow$ Embedded systems; Redundancy; Robotics; • Networks $\rightarrow$ Network reliability;

\section{KEYWORDS}

Coverage, Estimate, Healing, Hybrid sensor networks

Permission to make digital or hard copies of all or part of this work for personal or classroom use is granted without fee provided that copies are not made or distributed for profit or commercial advantage and that copies bear this notice and the full citation on the first page. To copy otherwise, to republish, to post on servers or to redistribute to lists, requires prior specific permission and/or a fee.

QoE-RAMC 2017, July 13-14

Copyright @ 2017 EAI 978-1-63190-156-0

\section{INTRODUCTION}

With the development of the network technology and extending of applied range of wireless sensor networks(WSNs), wireless sensor networks have gained widely attention for applying in natural disasters and some dangerous environment [6]. For example earthquake stricken area and old-growth forest fire. Dangerous environment exits huge security risk for the rescuers if they need enter the primary scene, but by casting a mount of sensors to the under surveillant area can not only solve the security risk problem but also make up WSNs networks to obtain the instant information of disaster area. However, because of the sensors are randomly deployed and may lead to the coverage holes, and as the data-centered network how to achieve efficient coverage is a very worthy issue to research [4].

Ghosh et al. [2] proposed the COVEN algorithm to increase the coverage, they based on the Voronoi diagram to make accurate calculation about the coverage holes. The method divided the detecting field into many cells and then divided every cell into several triangles, next discussed the relationship between sensor radius and Voronoi edge $l_{i j}$, then worked out the uncovered area in every cell, finally dispatched the assisted sensors to heal the coverage holes. The position$\mathrm{s}$ of assisted nodes in COVEN algorithm must satisfy three conditions as follows:

- $P_{i}$, the positions of assisted nodes must be located on the line that bisects the inner angle formed by $V_{i}$, which is Voronoi vertex;

- $P_{i}$ is located in the Voronoi polygon that is constructed by current sensor nodes;

- The distance between sensor node $S_{i}$ and assisted node $P_{i}$ is the minimum value compared with double sensing radius, $d\left(S_{i}, P_{i}\right)=\min \left\{2 R_{s}, d\left(S_{i}, V_{i}\right)\right\}$.

Obviously, it could increase the coverage to some extent, but it did not give the concrete algorithm about how to work out the number of assisted sensors, in addition to this, the calculation of coverage hole is relatively complicated.

Our paper resemble the paper [7] in frame. The general idea is to detect the coverage holes in every triangle, then dispatch nodes to heal the holes. What is different from previous work mainly is the simpler divide approach. In this paper, we study the area coverage of hybrid networks [7]. Assuming the initial network deployed a certain number of static nodes in the sensing field stochastically. At first, we use 
static sensors to estimate the coverage holes in the random distribution sensor network, and then calculate the optimal position to deploy the assisted sensors which can heal the coverage holes. Compared with the existing methods, our approach mainly shows several advantages as follows.

- Our calculation method of coverage is simpler than other works, the simulation in section 4 shows that our approach outperform previous works by get a higher coverage ration.

- We give out the optimal position to heal the the coverage hole.

The rest of the paper is organized as follows. In Section 2 , the system model and problem statement is illustrated. Then, we propose the theoretical framework of the problem and our coverage algorithm in Section3. In section 4, performance evaluation and analysis are presented. Conclusions and future work are given in the last part Section 5.

\section{SYSTEM MODEL}

The WSNs are consisted by many mobile sensors and static sensors, the fundamental problem in this paper is how to enhance the coverage by finding and healing coverage holes. Next, we will introduce some preparative knowledge which are used in the following analysis.

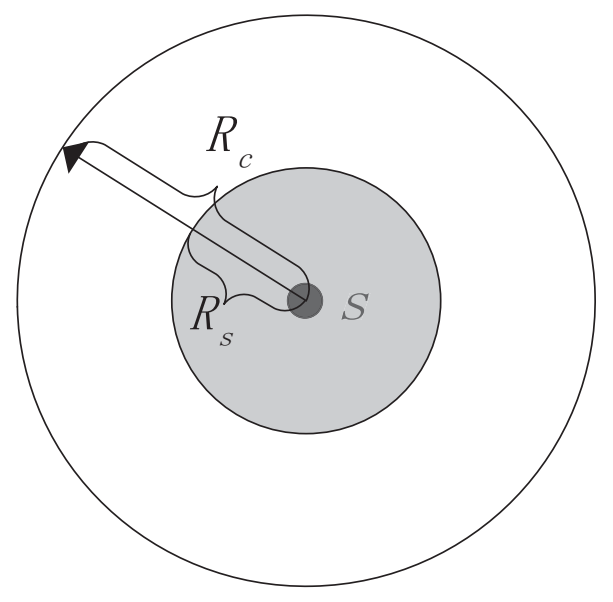

Figure 1: Communication and sensing models

\subsection{Communication and Sensing Model}

Each sensor has communication capacity and sensing capacity, $R_{c}$ is defined as communication radius and $R_{s}$ is defined as sensing radius, Figure 1 shows the communication and sensing models. If and only if the distance between two sensors is within $R_{c}$, they can communicate with each other, otherwise the node is isolated. Tian et al. [5] proved that $R_{c} \geq 2 R_{s}$ is the sufficient condition of tight lower bound to ensure network connectivity.
Delaunay triangulation is an important data structure in computational geometry [1]. The most significant property we use in this paper is that:

(1) Maximum empty circle characteristic. The property decides the Delaunay triangulation is unique and any four points must not construct a circle.

(2) Maximize the minimum angle. The characteristic mean$\mathrm{s}$ the composition of triangle is reasonable and not too narrow.

We can detect the coverage holes in each Delaunay triangle by discussing the $R_{s}$ and the circumcenter $C_{0}$ of the triangle. If $C_{0}$ is not covered by the sensor node of triangle, there will exist coverage holes in the triangle. Given $N$ static sensors $S_{1}, S_{2}, S_{3} \cdots$, we can get the Delaunay Triangulation (DT). For example, we choose 50 random nodes to construct Delaunay Triangulation in a $100 \times 100$ scene by MATLAB as shown in Figure 2.

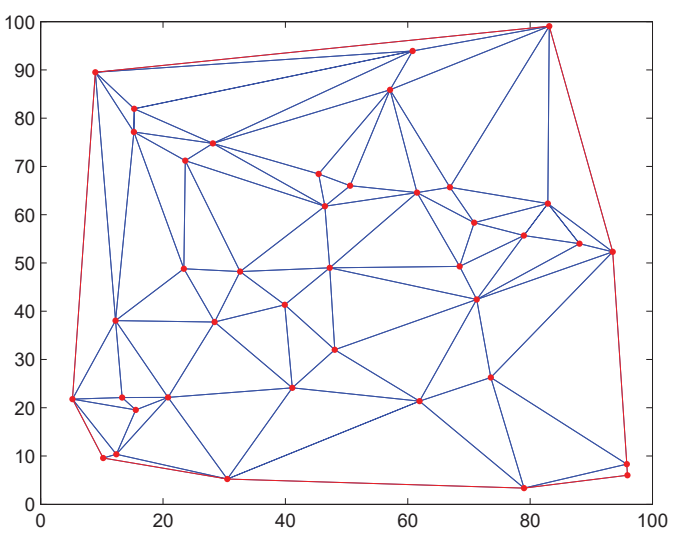

Figure 2: Delaunay Triangulation (DT)

\subsection{Problem Statement}

The coverage of the wireless sensor network can be divided into area coverage, point coverage and barrier coverage. In this paper we mainly discuss the scheme of position selected. At first, we assume a $100 m \times 100 m$ scene and deploy a certain number of static sensors randomly in this area. The problem we are prepared to address is how to choose the position to deploy healing sensor nodes. To solve this issue, we need to divide the scene into small cell by Delaunay Triangulation and then discuss the position of assisted node in each cell.

To simplify the analysis of the problem later, we assume that all sensors' sensing range is a circular with the radius of $R_{s}$, and $R_{c}$ is defined as communication range. In order to facilitate reading, we introduce some significant parameters as Table 1: 
Table 1: main notations.

\begin{tabular}{ll}
\hline Notation & Definition \\
\hline$S_{i}, M_{j}$ & Static sensor and mobile sensor \\
$\theta_{i}$ & Angle of three Delaunay triangle \\
$R_{s}, R_{c}$ & Sensing radius and communication radius \\
$P_{i}$ & Position of mobile assisted sensor \\
$C_{0}$ & The circumcenter of the $\Delta S_{1} S_{2} S_{3}$ \\
$S_{\text {uncovered }}$ & Uncovered area of the triangle \\
$d\left(s_{i}, s_{j}\right)$ & Euclidean distance between $s_{i}$ and $s_{j}$ \\
$\mu$ & Input parameter to round the number \\
& of assisted sensors \\
\hline
\end{tabular}

\section{COVERAGE HOLES DETECTING AND HEALING}

In this section, we will elaborate the problem we have proposed above and the Heal Coverage Holes Algorithm(HCHA). The network we research is hybrid, which consists of both static and mobile sensors. During the network initialization, static sensors construct the DT.

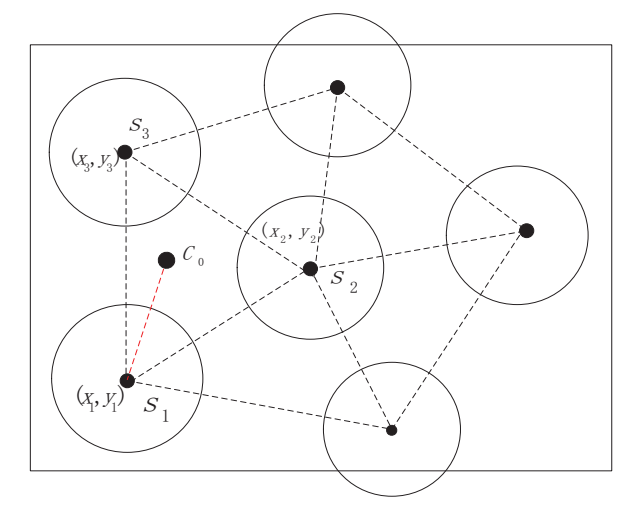

Figure 3: Nodes construct triangle.

Step1. Detect if there exist the coverage holes. The approach is based on the DT which can constructed by the static sensors at the beginning.

We assume a $100 m \times 100 m$ scene, in the scene senors are deployed randomly may cause the coverage holes. Helping with the DT, we can judge if there exist coverage holes or not. Nodes construct triangles as Figure 3 shows, $C_{0}$ is the circumcenter of the $\Delta S_{1} S_{2} S_{3}$ and the distance $d\left(S_{i}, C_{0}\right)$ is circumradius $R$. If $R_{s}>R$ then there does not exist coverage hole between the three sensors, if the $\Delta S_{1} S_{2} S_{3}$ is an acute triangle and $R_{s}<R$ then there must exist coverage holes between three sensors [3] .

We know $C_{0}$ is the circumcenter of $S_{i}(1,2,3)$, according to geometry we can get conclusion easily that the distance between $C_{0}$ and $S_{i}$ is equal, so if $C_{0}$ is covered by sensor $S_{1}$, then $C_{0}$ must be covered by $S_{1}^{\prime} s$ neighbour nodes $S_{2}$ and $S_{3}$ as the Figure 4(b) demonstrates. It is apparently that the
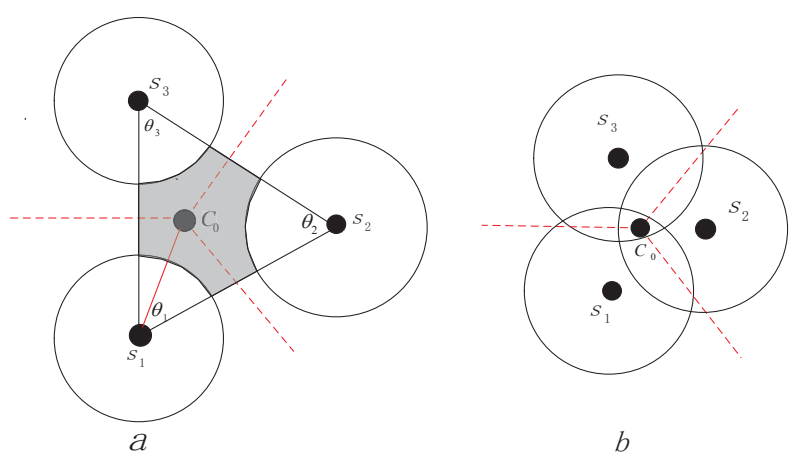

Figure 4: Coverage holes estimated.

triangle area constructed by $S_{1}$ and its two neighbours do not exist coverage holes.

Step2. Heal the coverage holes by finding the position of assisted nodes.

In order to heal the coverage holes efficiently, it is easy to satisfy the bow area as Figure 5 shows is minimum. We introduce two assisted angles $\alpha, \beta$ and $P_{0}$ which is the inner of $S_{\Delta p_{1} R_{1}^{1} R_{2}^{1}}$ to simpler the calculation. By mathematical theory we can get the area of $S_{\Delta p_{1} R_{1}^{2} R_{2}^{2}}$ and $S_{\text {sector } 2}$, and $S_{\text {sector } 1}$ :

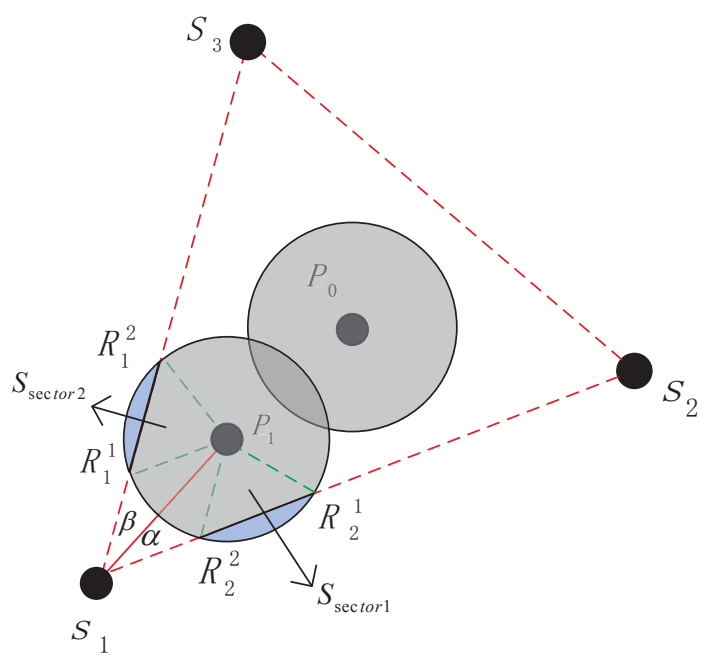

Figure 5: Node $P_{1}$ lies in the angle bisector.

$$
\left\{\begin{aligned}
S_{\Delta p_{1} R_{2}^{1} R_{2}^{2}} & =d \sin (\alpha) \sqrt{R_{s}^{2}-d^{2} \sin ^{2}(\alpha)} \\
S_{\Delta p_{1} R_{2}^{1} R_{2}^{2}} & =d \sin (\beta) \sqrt{R_{s}^{2}-d^{2} \sin ^{2}(\beta)} \\
S_{\text {sector } 1} & =\frac{R_{s}^{2}}{2}\left(\pi-2 \arcsin \left(\frac{d \sin \alpha}{R_{s}}\right)\right) \\
S_{\text {sector } 2} & =\frac{R_{s}^{2}}{2}\left(\pi-2 \arcsin \left(\frac{d \sin \beta}{R_{s}}\right)\right)
\end{aligned}\right.
$$




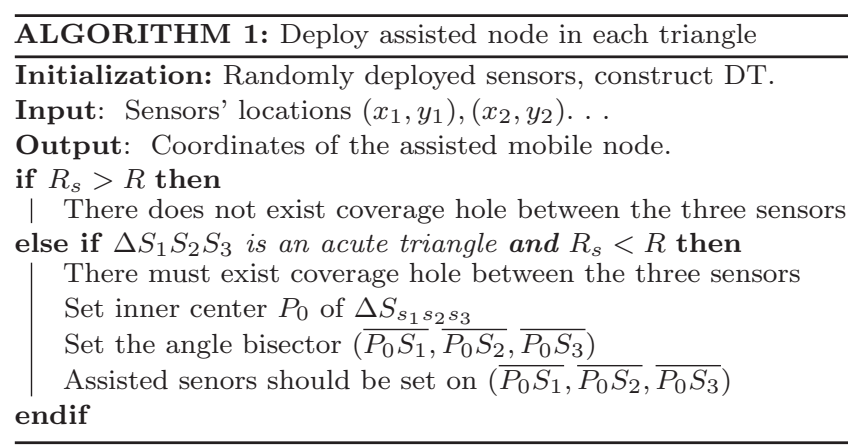

we can get the $S_{\overparen{R_{2}^{1} R_{2}^{2}}}=S_{\text {sector } 1}-S_{\Delta p_{1} R_{2}^{1} R_{2}^{2}}$, in a similar way $S \overparen{R_{1}^{1} R_{1}^{2}}$ can be calculated. Finally, the blue area is:

$$
\begin{aligned}
S_{\text {Bowarea }} & =S_{\overparen{R_{1}^{1} R_{1}^{2}}}+S_{\overparen{R_{2}^{1} R_{2}^{2}}} \\
& =\sum_{\phi=\alpha, \beta} \frac{R_{s}^{2}}{2}\left(\pi-2 \arcsin \left(\frac{d \sin \phi}{R_{s}}\right)\right) \\
& -d \sin (\phi) \sqrt{R_{s}^{2}-d^{2} \sin ^{2}(\phi)} .
\end{aligned}
$$

According to the extremum theorem of the two function , easily we can get when $\alpha=\beta, S_{\text {Bowarea }}$ is the least, thus $\angle S_{2} S_{1} P_{1}=\angle S_{3} S_{1} P_{1}$, so $\overline{P_{1} S_{1}}$ is the angular bisector of $\angle S_{2} S_{1} S_{3}$, then we prove that assisted sensor must lie on the angle bisector of $S_{\Delta} s_{i} s_{j} s_{k}$ which is constituted by three neighbor nodes.

Pseudo-code is given in ALGORITHM 1.

\section{PERFORMANCE EVALUATION AND ANALYSIS}

In this section, we will conduct comparative simulations about our algorithm HCHA and COVEN, the main simulation platform we used is Matlab. In order to make a remarkable contrast, we compare HCHA deployment with the random deployment and COVEN deployment in paper [2]. The simulation area we choose is $100 \mathrm{~m} \times 100 \mathrm{~m}$ and sensing radius $R_{s}$ is $5 \mathrm{~m}, \mu$ is 0.5 , red nodes represent mobile sensors and blue represent static sensors.

As shown in Figure 6 the coverage ratio of HCHA deployment is higher than both random deployment and COVEN deployment. The HCAH deployment has a better performance when the number of total nodes is less than 120 , and when the the number of total nodes is between 120 and 240, the HCHA still has a higher coverage ratio than both of COVEN and random deployment, as to the number of total nodes higher than 240, the coverage ratio between $\mathrm{HCHA}$ and COVEN hold the line.

The Figure 7 has displayed the optimal position of assisted sensor. Three blue nodes represent the static sensors and they construct the DT which is displayed by the blue triangle. To illustrate the optimal position of assisted sensors which are used to heal the coverage holes, we construct the DT with coverage holes at the beginning. The red points represent the optimal position of assisted sensors, the red circles show

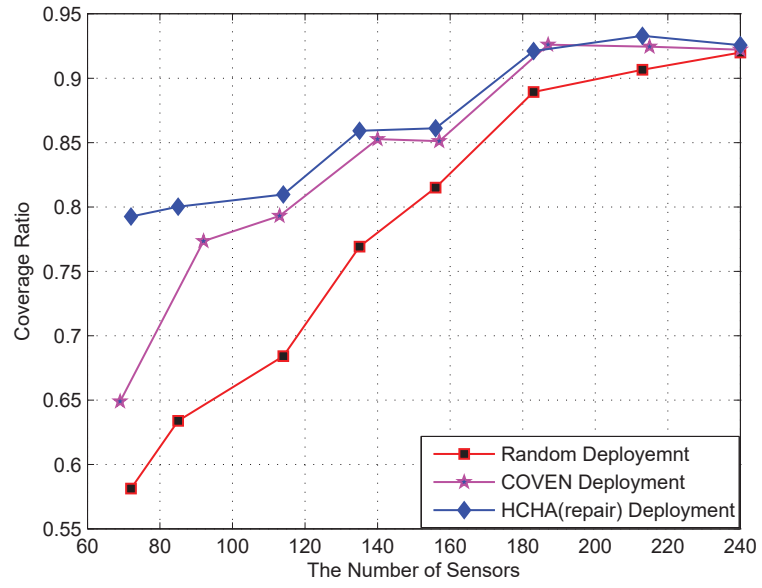

Figure 6: Coverage ratio.

the healed coverage holes. The optimal position of assisted sensors should be the angle bisector of three neighbor nodes as we have proved.

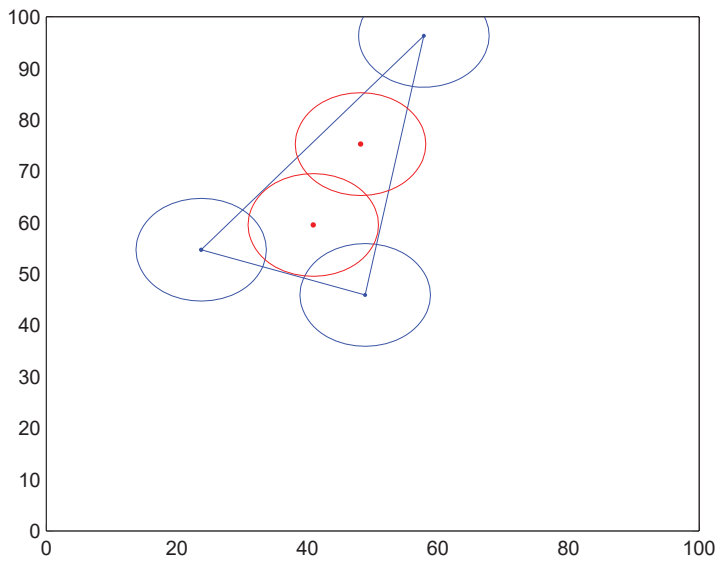

Figure 7: Optimal location of assisted sensor in HCHA.

\section{CONCLUSIONS AND FUTURE WORK}

In this paper, we study the coverage problem in hybrid WSNs and we give HCHA algorithm based on Delaunay to estimate as well as heal coverage holes in the monitoring area, besides we solve the boundary coverage holes estimation and consider multiple assisted nodes healing deployment strategy.

For the future work, we are prepared to research the quantitative range of assisted nodes which is related to parameter 
$k$, where $k$ is the ration between $R_{c}$ and $R_{s}$. In addition, we are going to research the detail healing method by collecting the neighbour sensor nodes information.

\section{ACKNOWLEDGMENT}

This work was supported in part by NSF of China under Grant 61301118 and Grant 71171045, in part by the International S\&T Cooperation Program of Shanghai Science and Technology Commission under Grant 15220710600, in part by the Innovation Program of Shanghai Municipal Education Commission under Grant 14YZ130, in part by the Fundamental Research Funds for the Central Universities, in part by the National Natural Science Foundation of China under Grant 61602110 and in part by the National Science Foundation for Postdoctoral Scientists of China under Grant $2016 \mathrm{M} 591575$.

\section{REFERENCES}

[1] Steven Fortune. 1992. Voronoi diagrams and Delaunay triangulations. Computing in Euclidean geometry 1, 193-233 (1992), 2 .

[2] Amitabha Ghosh. 2004. Estimating coverage holes and enhancing coverage in mixed sensor networks. In Local Computer Networks, 2004. 29th Annual IEEE International Conference on. IEEE, 68-76.

[3] Hwa-Chun Ma, Prasan Kumar Sahoo, and Yen-Wen Chen. 2011. Computational geometry based distributed coverage hole detection protocol for the wireless sensor networks. Journal of network and computer applications 34, 5 (2011), 1743-1756.

[4] Avinash More and Vijay Raisinghani. 2014. Random backoff sleep protocol for energy efficient coverage in wireless sensor networks. In Advanced Computing, Networking and InformaticsVolume 2. Springer, 123-131.

[5] Di Tian and Nicolas D Georganas. 2005. Connectivity maintenance and coverage preservation in wireless sensor networks. Ad Hoc Networks 3, 6 (2005), 744-761.

[6] Gurkan Tuna, V Cagri Gungor, and Kayhan Gulez. 2014. An autonomous wireless sensor network deployment system using mobile robots for human existence detection in case of disasters. Ad Hoc Networks 13 (2014), 54-68.

[7] You-Chiun Wang. 2014. A two-phase dispatch heuristic to schedule the movement of multi-attribute mobile sensors in a hybrid wireless sensor network. IEEE transactions on mobile computing 13, 4 (2014), 709-722. 